\title{
Ueber die Einwirkung von Phtalsäureanhydrid auf die secundären Monamine*)
}

\section{von A. Piutti,}

Assistent am Laboratorium $z$ u Florenz.

(Eingelaufen den 8. November 1884.)

Wie ich früher (Gazz. chim. 18, 329) mitgetheilt habe, spaltet sich die Phtalamidobenzoësäure mit primären Aminen im Sinn der algemeinen Gleichung :

$$
\begin{aligned}
& \mathrm{COOH} \cdot \mathrm{C}_{8} \mathrm{H}_{4} \cdot \mathrm{N}<\left\langle\mathrm{CO}>\mathrm{C}_{6} \mathrm{H}_{4}+\mathrm{NH}_{2} \cdot \mathrm{C}_{\mathrm{n}} \mathrm{H}_{\mathrm{m}}\right. \\
& =\mathrm{C}_{6} \mathrm{H}_{4}<\mathrm{CO}>\mathrm{C} \cdot \mathrm{C}_{\mathrm{n}} \mathrm{H}_{\mathrm{m}}+\mathrm{C}_{6} \mathrm{H}_{4}<\mathrm{COOH}
\end{aligned}
$$

Hieraus wird ersichtlich, dafs diese Säure, indem sie mit dem Amin das substituirte Phtalimid bildet, sich in ihrem Yhtalsäuretheil wie Phtalsãureanhydrid verhält. Ich habe daher zunāchst die Einwirkung des Phtalsäureanhydrids auf secundäre Monamine untersucht.

Nach der Theorie lassen sich für diese Reaction zwei Fâlle voraussehen; nämlich :

I. Dafs gleiche Molecule Anhydrid und Amin sich direot vereinigen, ohne Austritt von Wasser, und so eine disubstituirte Phtalaminsäure bilden :

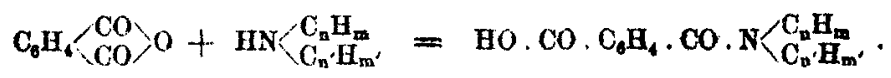

Il. Dafs 1 Mol. Anhydrid auf 2 Mol. Amin einwirkt, unter Austritt von Wasser, und dafs nun ein vierfach-substituirtes Derivat eines Phtaldiamids entsteht :

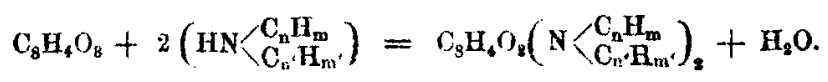

Mit den Basen, womit ich meine Versuche angesteft habe, Aellylanilin, Diphenylamin, Piperidin und Coniin, verlăuft die Reaction nach beiden theoretisch vorauszusebenden Richtungen.

*) Nach dem Original aberseint von Stephan Wortheim. 
In dem Mafse, als sich die substituirte Phtalaminsäure bildet, verbindet sie sich mit der noch nicht in Reaction getrelenen Base zu einem Salz, und dieses Salz giebt durch Einwirkung der Wärme, unter Austritt eines Moleculs Wasser, ein substituirtes Diamid.

Binvirkung von Phtalsäureanhydrid auf Aethylanilin.

Bekanntlich kann man die Alkylderivate, die aus dem käuflichen, wenn auch gereinigten Anilin dargestellt sind, durch fractionirte Destillation nicht von einander trennen; es bleiben ihnen immer Derivate von o- und p-Toluidin und selbst unangegriffenes Anilin und Toluidin beigemengt.

Ein schon mehrfach fractionirtes Aethylanilinpräparat theilte ich daher in zwei Theile, einen bei 203 bis $209^{\circ}$, einen zweiten bei 209 bis $215^{\circ}$ siedenden. Um aus diesen beiden Fractionen, welche immer noch Gemenge waren, die Verbindungen, die ich orzielen wollte, rein $\mathrm{zu}$ erhalten, benutzte ich ein Verfahren, das zugleich erlaubt, die einzelnen Constituenten des angewandten Aethylanilins in reinerem Zustand zu gewinnen.

Zu beiden Fractionen fügte ich Phtalsäureanhydrid hinzu und zwar etwa den zwanzigsten Theil der Menge, die man gebraucht hätte, um das Aethylanilin, letzteres als rein angenommen, vollständig in das substituirte Amid überzuführen. Ich erhielt hierauf die Lösung an Rückflufskühler auf einem Asbestbad im Sieden und fraciionirte dann. Die erst übergehenden Antheile enthielten das bei der Reaction entstandene Wasser. Das ferner übergehende wasserfreie Destillat unterwarf ich wieder der Einwirkung eines weiteren Zwanzigstels von Phtalsâureanhydrid a. s. w.

Jedesmal blieb im Destillationsgefärs ein häufig krystallinischer Rückstand, aus dem jich mil verdünnter Salzsăure 
noch jene geringe Menge freier Base auszog, die bei der Destillation nicht übergegangen war.

Durch diese Behandlung mit Salzsäure wurde der Rückstand fest; er wurde mit Wasser gewaschen, getrocknet, in Alkohol gelöst und durch fractionirte Krystallisation in die einzelnen Verbindungen zerlegt.

So kam ich zu folgenden Resultaten :

Von der Menge des Aethylanilins, dessen Siedepunkt bei 203 bis $209^{\circ}$ lag, erhielt ich durch Einwirkung je eines Zwanzigstels Phtalsäureanhydrid in der ersten Behandlung Phenylphtalimid; in der zweiten Phenylphtalimid mit wenig o-Tolylphtalimid; in der dritten 0-Tolylphtalimid mit wenig Phenylphtalımid und Aethylanilphtalein; in der vierten 0-Tolylphtalimid und Aethylanilphtalein; in der fünften, sechsten and siebenten Aethylanilphtalein, Aethyltoluidinphtaleïn.

Nach der letaten Behandlung schied sich das Aethylanilin durch fractionirte Destillation in zwei Theile, von denen der eine bei 204 bis $205^{\circ}$ siedete und fast reines Aethylanilin war, während der zweite, bei höherer Temperatur übergehende, zum gröfsten Theil aus tertiären Basen bestand und daher der folgenden Fraction zugefügt wurde.

Von diesem bei 209 bis $215^{\prime \prime}$ siedenden Theil des Aethylanilins erhielt ich bei Einwirkung eines Zwanzigstels Phtalsäureanhydrid 0-Tolylphtalimid, in der zweiten und dritten Behandlung Aethyltoluidinphtalein.

Nun blieb ein Diäthylanilin zurück, das bei 213 bis $215^{\circ}$ siedete, mit Oenanthol nur eine leichte Trübung gab und also nur Spuren secundärer Basen enthielt *).

*) Gerade un die Einwirkung der secunduren Basen auf Odannthol und andere Aldehyde zu studiren, hatte ich ror 16 Jahreu das Aethylanilin dargestellt, dessen letzte Reste bei der Arbeit des Dr. Piutti dienen sollten. Das Handolsanilin jener Zeit war bekanntlich eine noch viel complicirtere Misciaung, 
Im Folgenden theile ich zunächst die Resultate mit, die ich bei Einwirkung von Phtalsäureanhydrid auf gereingtes

als man dies nach den von Herm Piutti jetzt in dem Rück. atand des Aethylanilinprăparats gefundensn Körporn voraussetzan sollte.

Um diese äthylirten Basen darzustellen, bediente ich mich damals nur des wischen 183 and $185^{\circ}$ siedenden Theils des Anilins, aber ich besitze noch immer Fractiouen, welche boi und oberhalb $200^{\circ}$ sieden und die sich beuto nicht mehr im käuflichen Anilin rorfinden. Uim mich you den einzoluon Gemengtheilen der Mischung unabhlngig zu machon und um ein reines Derivat des Aethylanilins qu erhalten, ungeachtes äer Gegenwart einer geringen Menge anderer Basen, bediente ich mich schon demals derselben Methode der fractionirteu Reaction, wolche ich jetzt von Dr. Pi utti bei seiner obigon Arbeit habe snwenden lassev. Ich glaube, dafs diese Methode, dis mir durch den Gedanken an die fractionirio Präcipitation eingegebsa wurdo, mit Vortbeil unch in auderen zhulichen Fullen dienen kann.

Dio Linwirkung aromatischer Auine auf die Aldehyde, ohno Gegenwart waserentziohender Agentien, ist in Folge meiner Arbeiten aus den letzten 20 Jabren nach den verschiedensten Richtungen hin untersucht worden, und sie gilt als so charakteristisch für die Aldohyde, dafs mau in öftwron Fallen oin bezügliches Anilinderivat daretellt, wenn es sich darum handelt, dio Aldehydiunction irgend einer neaen Verbindung aschzuweisen. Eine der orsten Verbindungen, dio ich im Jahre 1864 daratellto, war des Anilinderivat des Oenanthols. Das Naphtalinderivat dieses Aldehyds wurde im Jahre 1873 von Papasogli dargestellt. Boi allen dioeon Feactionen reagirten Aldebyde und Amine unter Austritt von Wasser. Daher fiel es wir auf, dafs nouordings A. R. Leeds (Ber. d. deutech. chem. Ges. 10, 287, Januar 1883), wio os seheint ohno Kenntnifs der früheren Arbeiten, angiebt gefunden $q u$ hxben, dafs Anilin, Naphtalia und Xylidin sich direct mit Oenantiol reroinigen uad, ohne Ausscheidung ron Wsaser, aufserordentlich bewoglich Flüssigkeiten bilden, die sber nichts destoweniger nicht ohns /ersetzang destillirbar sind. Da ich noch keine Erfahrung in Betreff des Xylidins batte, unterwarf ich auch diese Base der Kinwirkung des Denanthols; anch in diesem Fallo verlief die Reaction auf gleicho Weise und unter Austritt yon Wasser, wie in allen anderen Fullen, zowohl weun ich nach don ron Lueds angegoboneu 
Aethylanilin erhielt, dann beschreibe ich die substituirten Amide, die ich aus der Behandlung der beiden Fractionen der gemischten Aethylaniline gewann, und behalte mir für das Ende dieser Abhandlung die Beschreibung des o-Tolylphtalimids vor, das bisher noch nicht beschrieben wurde.

\section{Aethylphenylphtalaminsäure.}

Man erhält diese Säure direct durch Auflosen eines Moleculs Phtalsäureanhydrid in 2 Mol. Aethylanilin. Es entsteht eine teigige, etwas gefärbte, dichroïstische Substanz, die man mit Salzsäure behandelt und mit Aether auszieht. Die ätherische lösung läfst wach dem Verdunsten die Säure fast rein zurück. Wendet man gieiçhe Molecule von Anhydrid und Amin an, so kommt man zu demselben Resultat, aber ein Theil des Phtalsäureanhydrids tritt dann nicht in Reaction.

Die Aethylphenylphtalaminsäure ist ein farbloses, erst nach längerer Zeit Krystalle abselzendes Oel, dichter als Wasser, in dem es etwas löslich ist; sehr löslich aber ist sie in den gewöhnlichen Lösungsmittelı Alkohol, Aeher u. s. w. Mit concentrirter Salzsäure zerfällt sie in Phtalsäure und das Chlorhydrat des Aethylanilins. Sie verbindet sich mit den alkalischen Basen zu in Wasser leicht löslichen Salzen.

Aethylphenylphtalaminsaures Kupfer. - Man löst die Aothylphenylphtalaminsäure in der genau nöthigen Menge von Ammoniak. Die nicht sehr concentrirte wässerige Lösung des Ammoniaksalzes versetzt man mit essigsaurem Kupfer, so

Bedingungen arbeitete, als auch, wenn jede Temperaturerhöhung vermieden wurde.

Es wäre wünschenswerth, dals Herr Loeds vor allem dio Bedingungen gerauer präcisirte, unter denen dio ron ihm angogebenon Producte der directen Vereinigung von Aldehyden mit primaren aromatischon Monaminen erhaiten werdeu können.

Huyo Schiff. 
186 Piutti, Einwirleung von Phtalsdureanhydrid

lange noch ein Niederschlag entsteht. Dieser wird abfiltrirt, gewaschen, geprefst, von neuem in Wasser suspendirt, wieder abfiltrirt und an der Luft getrocknet. Er ist ein himmelblaues Pulver, in kaltem Wasser etwas löslich; schmilzt man das Salz im Kölbchen, so erstarrt es beim Erkalten zu einer grünen harzigen Masse. In gleicher Weise verhalten sich auch andere in Wasser unlösliche oder wenig lösliche Salze dieser Säure, und im Allgemeinen die Salze aller der substituirten Phtalaminsäuren, die ich untersucht habe.

0,6197 g Kupfersalz, boi $90^{\circ}$ getrocknet, hinterliobsen beim Glähes $0,0825 \mathrm{CuO}$.

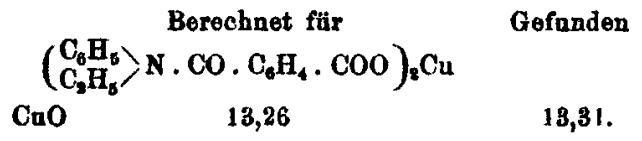

Dieses Salz verliert schon gegen $100^{\circ}$ Aethylanilin, schmilzt bei höherer Temperatur und zerfältt in Aethylanilin und Phenylphtalimid.

Aethylphenylphtalaminsaures Baryum. - Es wurde direct dadurch dargestellt, dafs man das Product der Reaction zwischen Phtalsäureanhydrid und Aethylanilin mit in Wasser suspendirtem kohlensaurem Baryum behandelte. Es ist in Wasser sehr löslich, löst sich auch in Alkohol, krystallisirt aber daraus nicht. Läfst man die wässerige Lösung langsam im Vacuum verdunsten, so bleibt eine glasähnliche oder weifsopake Substanz zurück, die leicht Aethylanilin verliert und sich in phtalsaures Baryum umwandelt.

Aethylphenylphtalaminsaures Silber wird in käsigen Klümpchen ans einer Lösung von äthylpbenylphtalaminsiaurem Ammoniak mit Silbernitrat erhalten. Es schwärzt sici sehr schnell am Licht.

Aethylphenylphtalamirsaures Aethylanilin. - Das durch Auflösen von Aethylanilin in Aethylphenylphtalaminsäure erhaltene Salz krystallisirt nicht. Im Vacuum über Schwefel- 
säure wurde es nach langer Zeit zu einer harzigen Masse, die sich mehr und mehr färbte. Es ist sehr löslich in Alkohol, weniger in Aether und in Wasser, in letzterem kalt mehr als warm.

Für die weiteren Umsetzungen bereitete ich eine gröfsere Menge dieses Salzes dadurch, dafs ich 2 Mol. Aethylanilin in wenig Alkohol löste und 1 Mol. Phtalsăureanhydrid hinzufügte. Die alkoholische Lösung warde verdunstet und der Rückstand mit etwas Wasser wieder aufgenommen; aus dieser Lösung liefs sich mit Aether etwas Aethylanilin ausziehen, das nicht in Reaction getreten war. Bei der Verdunstung des wässerigen Theils indessen erhielt man das Salz des Aethylanilins, durch alle seine Eigenschaften als solches charakterisirt.

Das äthylphenylphtalaminsaure Aethylanilin :

$$
\mathrm{C}_{6} \mathrm{H}_{4} \backslash \mathrm{COOH} . \mathrm{NH} . \mathrm{C}_{8} \mathrm{H}_{5}, \mathrm{C}_{6} \mathrm{H}_{5}
$$

verliert, auf $200^{\circ}$ erhitzt, 1 Mol. Wasser und geht in eine feste Verbindung über von der Zusammensetzung :

$$
\mathrm{C}_{8} \mathrm{H}_{4} \mathrm{O}_{2}\left(\mathrm{~N} . \mathrm{C}_{2} \mathrm{H}_{5} \cdot \mathrm{C}_{6} \mathrm{H}_{5}\right)_{2} \text {, }
$$

die nicht ein Derivat des normalen Diamids der Phtalsäure ist, sondern als ein Derivat des asymmetrischen Diamids, als Phtalein des Aethylanilins betrachtet werden mufs.

$$
\text { Aethylanilphtaleïn. }
$$

Das durch die Einwirkung der Wärme auf äthylphenylphtalaminsaures Aethylanilin erhaltene Product wurde mehrmals aus Aether krystallisirt. So gewann man sehr schöne, prismatische, glänzende Krystalle, die gegen $140^{\circ}$ schmelzen. Sie sind mit dem Product identisch, das man bei den letzten Behandlungen des bei 203 bis $209^{\circ}$ siedenden Aethylanilins rnit Phtalsäureanhydrid erhielt.

Wiederholt aus Alkohol umkrystallisirt, schmilzt das Aethylanilphtalein bei 140,5 bis $141,5^{\circ}$. Es ist unlöslich in 
Wasser, löslich in warmem Alkohol, in Aether und Benzin. Aus der atherischen Lösung kann man es bei langsamer Verdunstung in grofsen einige Centimeter langen Krystallen erhalten. Diese Krystalle zeigen eine charakteristische Zwillingsform; einzein sind sie in ihrer Form dem Colestin ährilich.

Aethylanilplitalein ist in verdünnter oder concentrirter wässeriger Kalilauge unlöslich; in alkoholischer löst es sich, ohne dadurch selust bei lungem Erhitzen zersetzt zu werden. Beim Schmelzen mit Kali hingegen zeriegt es sich leicht und vollständig in Aethylanilin und Phtalsäure. Auf $300^{\circ}$ erhilat spaltet es sich theilweise, aber es bijdel sich zurück und setat sich krystallinisch auf den kalten Siellen der Kolbenwand an. Der aus Alkohol krystallisirte Rückstand giebt wieder die ursprüngliche Substanz.

Ein aus Aether krystallisirtes und bei $100^{\circ}$ getrocknetes Präparat wurde der Analyse unterworfen.

$0,2305 \mathrm{~g}$ Substanz gaben 0,65s $\mathrm{CO}_{2}$ und $0,1348 \mathrm{H}_{8} \mathrm{O}$.

\begin{tabular}{|c|c|c|}
\hline & $\mathrm{C}_{2} \mathrm{~F}_{b}$ & Gefunten \\
\hline $\mathrm{c}$ & 77,41 & 77,40 \\
\hline H & 6,45 & 6,49 . \\
\hline
\end{tabular}

Aethylloluidinphtaloin.

Bei der zwetten und dritten Pehaudlung les bei 209 bis $215^{\circ}$ siedenden Fractionstheils der gemischten Aethylaniline mit Phtalsäureanhydarid und aus den altibholischen Mutterlaugen des Aethylanilphtaleins, das bei Behandlung des bei 203 bis $209^{\circ}$ siedenden Theils mit Phtalsäureanhydrid entstand, erhielt ich eine Substanz, die nicht krystallisiren wollte, auch nicht aus verschiederion Lösungsmitteln.

Nach Vertreibung des Alkohols aus der alkoholischen Lösung blieb eine amorphe, durchsichtige, feste und etwas gefärbte Substanz zuruck, die selir leicht schmolz, sich in Salzsäure und concentrirter Kalilauge nicht löste und die ich 
durch mehrmaliges Behandeln mit diesen Reagentien und schliefslich durch Erschöpfen mil Aether reinigte.

Die Analyse ergab folgende Werthe :

$0,2795 \mathrm{~g}$ gaben $0,7982 \mathrm{CO}_{8}$ und $0,1758 \mathrm{H}_{8} \mathrm{O}$.

\begin{tabular}{|c|c|c|}
\hline \multicolumn{3}{|c|}{$\begin{array}{c}\text { Berechnet für } \\
\mathrm{C}_{8} \mathrm{H}_{4} \mathrm{O}_{8}\left(\mathrm{~N}, \mathrm{C}_{8} \mathrm{H}_{\mathrm{E}} \cdot \mathrm{C}_{7} \mathrm{H}_{7}\right)_{7}\end{array}$} \\
\hline C & 78,0 & 77,88 \\
\hline H & 7,0 & 6,98 \\
\hline
\end{tabular}

Sehr wahrscheinlich ist diese Verbindung das Phtalein des Aethyl-0-toluidins; denn da es sich hier um eine secundäre Aethylbase handell, so bleiben, nach Abzug des Aethyls, für das andere Radical nur sieben Kohlenstofiatome, und so kann dieses also nur Toluyl sein. Folglich ist das mit dem Phtalyl verbundene Amin ein substituirtes Toluidin. Schliefst man das m-Toluidin aus, das sich im käullichen Anilin nicht findet, so bleiben nur o- und p-Toluidin zu berücksichtigen.

Da ich nun bei den Behandlungen des ersten Fractionstheils der gemischten Aethylaniline mit Phtalsäureanhydrid das o-Toluylphtalimid frei von p-Verbindung erhielt, so ist es fast gewifs, dafs auch das Aethyltoluidin, dessen Derivat das erhaltene Phtaleïn ist, die isomere o-Verbindung, und daher das Phtaleïn Aethyl-o-toluidinphtaleïn ist.

In 'der That erhielt ich, als ich diese Substanz mit Kali schmolz und destillirte, eine Base von dem Geruch des Geraniums; sie siedete bei 211 bis $215^{\circ}$, gab die Ho fm a n n'sche Carbylaminreaction nicht, charaliterisirte sich dagegen durch alle ihre Eigenschaften als ein secundäres Monamin.

In der Literatur findel sich für ein Aethyltoluidin der Siedepunkt 217 bis $218^{\circ}$ angegeben (Morley und A bel 1854), doch bezieht er sich gewils auf tine Mischung ron o- und p-Toluidin.

Es ist auffallend, dafs das Phtaleïn des Aethyltoluidins nicht krystallisiren will, wo hingegen das Phtaleïn des Aethylanilins gut krystallisirt. Diese Erscheinung mōchte vielleicht 
ihre Erklärung in der Anwesenheit einer geringen Menge einer anderen Substanz finden, welche durch Lõsungsmittel nicht davon getrennt werden konnte.

\section{Diphenylphtalaminsäure.}

Gleiche Molecule Diphenylamin und Phtalsäureanhydrid schmilat man in einem Kolben auf einem Asbestbad zusammen. Man lärst die Masse langsam bis auf $250^{\circ}$ erkalten und erhält sio etwa eine Stunde auf dieser Temperatur.

Beim Erkalten erstarrt das Ganze zu einer grünbraunen Masse, die aus diphenylphtalaminsaurem Diphenylamin besteht und mit Nadeln von Phtalsäureanhydrid durchsetzt ist. Man löst diese Substanz in wenig warmem Alkohol auf und fügt wässeriges Ammoniak hinzu. Das Diphenylamin wird so frei und scheidet sich theils beim Erkalten, theils beim weiteren Verdunsten aus. In der wässerigen Lösung bleiben diphenylphtalaminsaures und phtalsaures Ammoniak. Diese Lösung verliert beim Eindampfen Ammoniak und scheidet gelbe Krusten von Diphenylphtalaminsäure aus, während man aus den sehr concentrirten Mutterlaugen durch $Z$ usatz von Wasser eine weitere Menge derselben Säure gewinnt. Aus den letzten Mutterlaugen erhält man den Rest der Säure durch Fällen mit Salzsäure, Erwärmen des Niederschlags mit Wasser und schliefsliche Behandlung mit Aether, welcher etwas Phtaläther beseitigt, der sich bei diesen verschiedenen Operationen bildet.

Die wiederholt aus Alkohol krystallisirte Diphenylphtalaminsäure zeigt sich nun in Form von grofsen, weifsen und harten Warzen, oder kleinen glänzenden, dazwischen gelagerten Prismen. Sie schmilzt bei 147 bis $148^{\natural}$, ist unlöslich in Wasser, wenig löslich in Aether, sehr löslich in Alkohol.

Bei der Analyse eines zweimal aus Alkohol umkrystallisirten Prāparats ergaben sich folgende Resultate : 
$0,2500 \mathrm{~g}$ gaben $0,691 \mathrm{CO}_{2}$ und $0,108 \mathrm{H}_{2} \mathrm{O}$.

\begin{tabular}{|c|c|c|}
\hline & $\begin{array}{l}\text { chnet für } \\
\text { YoN }\left(\mathrm{C}_{8} \mathrm{H}_{6}\right)_{\mathrm{t}} \\
\mathrm{OOOH}\end{array}$ & Gefunden \\
\hline ( & 75,71 & 75,36 \\
\hline & 4,73 & 4,8 \\
\hline
\end{tabular}

Diese Sãure löst sich leicht in Alkalien und wird aus ihren Lösungen durch starke Säuren niedergeschlagen. In Schwefelsäure ist sie in der Kälte löslich, aber durch Zusatz von Salpetersäure erbält man keine Diphenylaminreaction; mit Wasser niedergeschlagen nimmt sie bald eine gelbe Färbung an.

Diphenylphtalaminsaures Silber. - Dieses Salz erhält man aus diphenylphtalaminsaurem Ammoniak mit Silbernitrat. Es ist ein krystallinisches, in Wasser etwas lösliches Pulver. Aus wässeriger, nicht erwärmter und in Dunkeln gehaltener Lõsung sotzt es sich nach einiger Zeit in weilsen Warzen ab, die sich aber am Licht sehr schnell schwärzen.

$0,311 \mathrm{~g}$ hinterliefsen $0,079 \mathrm{Ag}$, also $25,40 \mathrm{pC}$, während die Formel $\left(\mathrm{C}_{6} \mathrm{H}_{6}\right)_{2} \mathrm{~N}$. CO $\mathrm{C}_{6} \mathrm{H}_{4}$. COOAg 25,49 pC. verlangt.

Diphenylphtalaminsaures Kupfer ist ein Pulver von himmelblauer Farbe, das man durch Mischung einer Lösung des Ammoniaksalzes mit essigsaurem Kupfer erhält.

Durch doppelte Umsetzung gewinnt man auch das Bleisalz als einen weifsen, in Wasser unlöslichen Niederschlag. Ebenso erhält man das Baryumsalz, das sich aus einer Lösung von diphenylphtalaminsaurem Ammoniak mit Baryumchlorid nach einiger Zeit in sphärischen Warzen absetzt.

Diphenylphtalaminsaures Diphenylamin. - Die Diphenylphtalaminsäure giebt durch Schmelzen mit 1 Mol. Diphenylamin das diphenylphtalaminsaure Diphenylamin, eine feste, gefärbte, in Aether lösliche, aber daraus nicht krystallisirende Substanz. Lange Zeit auf $300^{\circ}$ erhitzt verliert sie nach und nach Wasser, wobei sie sich stark braun färbt und in das entsprerisende Phtalein übergeht. 


\section{Diphenylaminphtaleïn.}

Man erhält diese Verbindung direct durch meh̉rstündiges Kochen von Phtalsüureanhydrid (1 Mol.) mit Diphenylamin (2 Mol.) am Rückfluishühler, so lange als man Entwicklung von Wasserdampf beobachtet.

Das Reactionsproduct ist eine feste, stark gefärbte, besonders in warnem Aether, in Benzin und Alkohol lösliche Substanz. Aus der concentrirten ätherischen Lösung scheidet sich eine Kruste wenig gefärbter, durchsichtiger Krystalle ab, die sich durch Unkrystallisiren aus Alkohol, in welchem sie in der Kälte wenig löslich sind, reinigen lassen und die aus Diphenylaminphtaleïn bestehen. Diese Substanz krystallisirt aus Alkohol in langen, sternförmig gruppirten Nadeln, die völlig weifs und glänzend sind und bei 238 bis $238,5^{\circ}$ schmelzen. Aus Aether oder Benzin krystallisirt sie in starken Prismen.

$0,250 \mathrm{~g}$ Substanz gaben $0,7505 \mathrm{CO}_{8}$ und $0,120 \mathrm{H}_{8} \mathrm{O}$.

$$
\begin{array}{ccr} 
& \text { Berechuet für } & \text { Gefunden } \\
& \mathrm{C}_{8} \mathrm{H}_{4} \mathrm{O}_{9}\left[\mathrm{~N}_{\mathrm{N}}\left(\mathrm{C}_{6} \mathrm{H}_{6}\right)_{2}\right]_{2} & \\
\mathrm{C} & 82,05 & 81,84 \\
\mathrm{H} & 5,13 & 5,33 .
\end{array}
$$

Das Diphenylaminphtaleîn löst sich in wässeriger Kalilauge nicht. Schmilat oder kocht man es mit concentrirtem Kaii, so zerfällt es in Diphenvlamin und Phtalsäure. Es löst sich in concentrirter Schwefelsäure; fügt man zn dieser Lösung einen Tropfen Salpetersäure, so entsteht eine prächtig violetto Färbung, die auf Zusatz von Wasser wieder verschwindet. Es setzt sich jetzl eine braune Sulstanz $a b$, welche sich in Schwefelsäure wieder mit der angegebenen Färbung auflöst.

Die schwefelsaure Lösung des Diphenylamins zeigt bei gleicher Behandlung eine intensiv himmelblaıe Färbung.

Das Diphenylaminphtaleïn verwandelt sich bei Bebandlung mit concentrirter Salpetersäure in eine Nitroverbindung, 
die in Alkohol löslich ist und daraus in golben Blättchen krystallisirt. In Ammoniak färbt sie sich intensiv roth. Die Kaliverbindung ist blutroth, krystallisirt aber selbst aus sehr concentrirter Alkohollösung nicht.

Das Diphenylaminphtaleïn ist bereits bekannt. E. Lellmann*) hat dasselbe durch Einwirkung von Chlorphtalyl auf Diphenylamin erhalten, aber über dessen Constitution sich nicht ausgesprochen.

Die Eigenschaften der nach den beiden verschiedenen Methoden erhaltenen Substanzen sind die nämlichen. Setzt man für das Chlorid des Phtalyls die von Ador **) vorgeschlagene und durch v. Gerichten bewiesene Formel, in der die beiden Chloratome an dasselbe Kohlenstoffatom gebunden sind :

$$
\begin{aligned}
& \mathrm{C}_{6} \mathrm{H}_{4} \cdot \mathrm{C}=\mathrm{CH}_{2} \\
& 1 \quad 1 \\
& \mathrm{CO}-\mathrm{O}
\end{aligned}
$$

so müssen wir auch für die Verbindung des Diphenylamins, die man mittelst Phtalylchlorid oder Pbtalsäureanhydrid erhält, dieselbe asymmetrische Constitution annehmen; und so kamn man daher die Constitulion der im Vorhergehenden beschriobenen Phtaleine in der allgemeinen Formel wiedergeben :

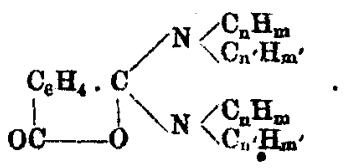

Die Constitution dieser Verbindungen giebt zugleich die Erklärung, warum sie nicht die charakteristischen Eigenschaften dor wirklichen Amide haben ***).

*) Ber. d. deutsch. chem. Ges. 15, 830.

*) Daselbst $18,323$.

**) Neben dem Diphonylaminphtaleïn bildet aich eine in gelben Prismen krystallisirende, bei 256 bie $258^{\circ}$ schmelzende $Y$ orbindung; sie findet sich in den letzten a!koholischen Muttorlangen von der Anasion der Cotomia 397 . Bd. 
Wie äthylphtalaminsaures Aethylanilin und diphenylphtalaminsaures Diphenylamin in der Wärme Wasser abspalten und die entsprechenden Phtaleine liefern, so kōnnte man durch Einwirkung der Wärme auf das diphenylphtalaminsaure Aethylanilin zu einem azotirten gemischten Phtaleïn gelangen. Ich habe daher Diphenylphtalaminsäure in einem geringen Ueberschufs von Aethylanilin aufgelöst und diese Lösung einige Zeit zum Sieden erhitzt. Sie färbte sich dabei stark nnd Diphenylamin wurde ausgeschieden. Es bildete sich äthylphenylphtalaminsaures Diphenylamin, das mit Salzsăure in Aothylphenylphtalaminsäure und Diphenylamin zerfiel.

Erhitzt man das äthylphenylphtalaminsaure Biphenylarnin lange und bis zu höherer Temperatur, so erhält man derartig gefărbte Producte, dafs sie zu einer weiteren Untersuchung nicht einladen.

Piperilenphtalaminsäure.

Ich liefs anfangs für die Reaction von Phtalsäureanhydrid auf Piperidin gleiche Molecule auf einander wirken. Das Phtalsäureanhydrid wurde dem Piperidin allmählich zugesetzt; die Renction ist von starker Wärmeentwicklung begleitet, so dafs man wohl thut abzukühlen, um kein Piperidin zu ver-

Reinigung jener Substanz, konnte jedoch der geringen Monge wogen nicht weiter verfolgt werden. Ebenso befindet aich in

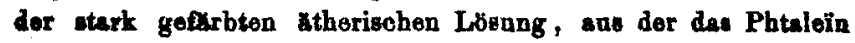
krystallivirte, sufeor diphonylphtslaminsaurem Diphenylamin eino Bubotenz, die in saurer Lösung eine dem Resorcinphtaleïn von Baejer tholiche Fluorescenz besitzt, indeseen durch Hinzufügen ron Alkali die Farbo verandert und oine sohr schöno azurblane Muorescenz annimmt, thnlich der einer Lösang von zweifachshwofelsaurem Chinin.

Bei Gelegerheit der Darstellung einor nouen Monge ron Dipbonylaminphtaleïn zum Zweck der Lösung anderer Fragen, dio sich auf den beweglichen Sauerstof der Phtalgruppe boaiehen, beabsichtige ich suf diese submtanzen surücksakommen 
lieren. Zu Ende der Reaction ist eine Menge völlig weifser, durchsichtiger, in Wasser und Alkohol sehr, in Aether wenig löslicher Krystalle entstanden.

Die wăsserige Lösung dieser Krystalle giebt bei Bebandluag mit 20 procentiger Kalilauge in der Kälte Piperidin $a b$; mit essigsaurem Kupfer liefert sie einen hellgrünen Niederschlag, also ein Anzeichen für die Gegenwart einer substituirten Phtalaminsäure *). Mit Salzsäure giebt sie ein farbloses schweres Oel, das Piperifenphtalaminsäure ist. Da sich sonach die Piperilenphtalaminsäure, kaum entstanden, mit dem Piperidin zu einem Salz verbindet, so mufs bei ihrer Bildung ein Theil des Phtalsäureanhydrids unangegriffen geblieben sein

Ich machte mir de Unlöslichkeit des piperilenphtalaminsauren Piperidins in Aether zu Nutzen und behandelte das Reactionsproduct nit diesem Lösungsmittel; aber das Salz nimmt mit solcher Begierde Wasser auf, dafs es bald durch Entwässerung des angewandten Aethers teigig wurde. Ich fügte nun eine kleine Menge Wasser hinzu, um es vollständig zu lösen und behandelte die so gewonnene Lösung wiederholt mit Aether.

Die Phtalsäure, welche sich aus dem Anbydrid bildet, tritt nicht in Reaction.

Nachdem die Piperilenphtalaminsäure aus ihrem Salz abgeschieden ist, nimmt sie Aether vollständig aus der wässerigen Lösung auf. Durch Veridunstung der ätherischen Löseng erhält man einen öligen Rückstand, der fast ganz aus Piperilenphtalaminsäure besteht, während im Wasser saures phtal-

*) Man darf diesen Niederschlag nicht mit dem hellgrünen Niederschlag von Kupferhydrexyd verwechseln, den Piperidin in einer Kupferlösnng giebt. Letzterer wird beim Erwkrmen scbwarz, aber das piperilenphtalaminsauro Kupfer schmilzt zu einer grünen harsigen Masse. 
saures Piperidin zarückbleibt, das, selbst lange Zeit im Vacuun über Schwefelsăure gehalten, nicht krystallisirt.

Die Piperilenphtalaminsäure, $\mathrm{HO} . \mathrm{CO}, \mathrm{C}_{6} \mathrm{O}_{4} . \mathrm{CO} . \mathrm{NC}_{5} \mathrm{H}_{10}$, is ein dickes farbloses Oel, in Wasser etwas, in Alkohol sehr löslich. Sie bildet mit den Alkalien leicht lösliche Salze and wird aus ihren Lösungen durch eine starke Säure niedergeschlagen. Durch weitere Einwirkung von Salzsäure wird sie in Piperidinchlorbydrat und Phtalsäure zerseizt.

Piperilenphitalaminsaures Kupfer erbäll man aus dem Ammoniaksaiz mit essigsaurem Kupier als hellblauen Niederschlag, der gewaschen, filtrirt, ausgeprefst und bei $95^{\circ} \mathrm{ge-}$ trocknet wurde.

$0,3796 \mathrm{~g}$ gaber beim Glulien 0,0565 Cuo.
Bereshnet für $\left(\mathrm{C}_{8} \mathrm{H}_{20} \mathrm{NCOC}_{6} \mathrm{H}_{4} \mathrm{CO}_{z}\right)_{2} \mathrm{C}:$ CuO is, 0 ?
Getunden 14,88 .

Das saure phtalsaure Piperidin, das man bei der vorhergehenden Reaction neben der Piperilenphtalaminsäure erhält, verlierl durch Erhitzen 1 Mol. Wasser und geht so in die gleiche Piperilenphtalaminsäure über, wie folgendes Schema verdeutlicht :

$$
\mathrm{C}_{8} \mathrm{H}_{4} \backslash \mathrm{COOH} . \mathrm{HNC}_{8} \mathrm{H}_{10}=\mathrm{C}_{5} \mathrm{H}_{4} \backslash \mathrm{COOH}_{6} \mathrm{CONO}_{40}+\mathrm{H}_{8} \mathrm{O}
$$

In Folge dessen wurde eme Portion dieses Salzes in einem Kölbchen auf dem Schwefelsäurobad erhitzt. Dic Entwicklung von Wasser begann obertialb $100^{\circ}$ und ging bis gegen 200" fort. Bei dieser Temperatur begann Phtalsäure zu subliniren. Jelzt wurde die Operation unterbrochen; die Masse erstarrte beim Erkalten.

Nach Neutralisation eines Theils mit Ammoniak erbielt man durch essigsaures Kupfer einen Niederschlag von piperilenphtalaminsaurem Kupfer und konnte auch mit Aether eine kleine Menge Piperileuphtalaminsäure ausziehen. Setzte man aber das Erhitzen über $200^{\circ}$ fort, so nahn die Menge des 
sublimirenden Phtalsäureanhydrids za und die Piperilenphtataminsäure zersetzte sich vollständig.

Aether zieit nun aus der Masse eine ölige, in concentrirtem und warnem Kali unlösliche Substanz aus, welebe die Bigenschafteit eines azotirten Pbtaleins zeigt.

\section{Piperilenaminphtalein.}

Dieses Phtalein erhielt ich aus Piperidinphtalat, von dem ich noch etwas zu meiner Verfügung hatte. Ich erhitzte dasselbe eine Stunde auf $210^{\circ}$ und hörte mit dem Erhitzen auf, als kein Phtalsäureanhydrid melir sublimirte. Das entstehende, etwas gefärbte und teigige Product wurde mit einer wässerigen concentrirten Kalilösurg behandelt. Durch Abkühlen mil Eis scheidet sich das Phtaleün aus der Kalüösung als eine etwas consistente Masse aus. In Aether gelöst wird es noch einmal der gleichen Behandlung unterworfen, um sicher alle Phtntsäure zu entiernen.

Nach Verdunstung der atherischen Lossung erliäl man das Phtalein als ein fast farbloses $0 \mathrm{el}$, welehes sehr allmählich zu farblosen Nadeln erstarrt, in den gewöhnlichen Mittein lostich ist, in der Kälte auch in vielem wässerigem Kalh. Durch Brwărmen der Kalilōsung wird es theilweise ausgeschieden.

Ich habe mit Sorgfalt die Zersetzung durch Schmeizen mit Kali wiederbolt und von neupro Piperidin exhalten, das frei wurce, und phtalsaures Kali, das in der Schmelamasse blieb. Die aus dieser Masse gewonnene Phtalsaure wurdo in Phtalsäureanhydrid und in Pheny!phtalinid verwandelt *\%.

-) Bei dieser Gelegexbsit priffí ich dag Verhalten du Fhbalsăure gegen geochmolzenes Xuli. Bei passendeza Frbitzen blekt vie zam gäroten Theil uangegriften; nur oine kloine Monge wird zerlegt und orthoilt dar gesoimolacann Masse oino purporne Farbung, walche durch Bebsndlung mit Wasser varschrindet. 
Zur Analyse wurde das so gewonnene Piperilenaminphtaleïn in einem Trockenschrank auf $80^{\circ}$ erwärmt und in das Vacuum über Schwefelsäure gebracht, bis es nicht mebr an Gewicht verlor.

0,258 g gaben $0,6805 \mathrm{CO}_{2}$ und $0,186 \mathrm{H}_{8} \mathrm{O}$.

$0,466 \mathrm{~g}$ gaben $40,2 \mathrm{cbcm} N$ bei $22,5^{\circ}$ und $758 \mathrm{~mm}$ Druck, also $37 \mathrm{cbcm} N$ bei $0^{\circ}$ und $760 \mathrm{~mm}=0,04647 \mathrm{~g} N$.

$\begin{array}{ccc} & \text { Berechnet für } & \text { Gefunden } \\ & \mathrm{C}_{3} \mathrm{H}_{4} \mathrm{O}_{4}\left(\mathbf{N} . \mathrm{C}_{\mathbf{6}} \mathbf{H}_{\mathbf{1 0}}\right)_{2} & \\ \mathrm{C} & \mathbf{7 2 , 0 0} & \mathbf{7 1 , 9 3} \\ \mathrm{H} & 8,00 & \mathbf{8 , 0 5} \\ \mathrm{N} & \mathbf{9 , 3 3} & \mathbf{9 , 9 9}\end{array}$

Die Umwandlung des Piperidinphtalats in Piperilenaminphtaleïn kann man in der Gleichung ausdrücken :

$2 \mathrm{C}_{6} \mathrm{H}_{6}<\mathrm{COOH} \cdot \mathrm{HNC}_{6} \mathrm{H}_{t 0}=\mathrm{O}_{8} \mathrm{H}_{4} \mathrm{O}_{2}\left(\mathrm{NC}_{8} \mathrm{H}_{10}\right)_{2}+\mathrm{C}_{6} \mathrm{H}_{4}<\mathrm{CO}>\mathrm{O}+3 \mathrm{H}_{2} \mathrm{O}$.

Rob. Schiff beschreibt *) ein Phtalylpiperid, welches er bei Einwirkung von 2 Mol. Piperidin auf $1 \mathrm{Mol}$ Phtalsüureanhydrid erhielt und zwar beim Arbeiten mit alkoholischen Lösungen. $\mathrm{Da}$ die auf diese Weise entstandene Verbindung eine grofse Aehnlichkeit mit meinem auf trockenem Weg dargestellten Präparat hat, habe ich die Reaction genau unter den von dem Autor angegebenen Bedingungen wioderholt. Dabei babe ich immer das gleiche Product erhalten, zu dem ich schon bei der Einwirkung gleither Molecule von Piperidin und Phtalsäureanhydrid ohne Zuthun von Alkohol gekommen war.

Die Verbindung, die sich aus alkoholischer Lösung durch die directe Vereinigung zweier Molecule Piperidin mit einem Molecul Phtalsäureanhydrid bildet, ohne Elimination von Warser, ist also nicht, wie angenommen, Phtalylpiperid, sondern das Piperidinsalz der Piperilenphtalaminsäure. Auch das von R. Schiff beschriebene Additionsproduct mit Brom pafst nach der Brombestimmung (gefunden 49,73 pC.) mehr zu einer Verbindung von vier Bromatomen mit piperilenphtalaminsaurem

*) 1งkzzz. Vol. 8, 33. 
Piperidin (berechnet $50,16 \mathrm{pC}$.), als zum supponirten Tetrabromphtalylpiperid (berechnet $51,61 \mathrm{pC}$.).

Nachden ich die Identität dieser beiden auf zwei Wegen dargesteliten Substanzen durch Vergleichung ibrer physikalischen Bigenschaften und ihres Verhaltens gegen Kali, Salasurure and essigsaures Kupfer genügend festgestellt hatte, habe ich noch aufserdem das Silbersalz unter Anwendung des aus der alkoholischen Lösung gewonnenen piperilenphtalaminsauren Piperidins dargestellt und analysirt.

Dieses Salz entsteht beim Hinzufügen von Silbernitrat zu einer wässerigen Lösung des piperilenphalaminsauren Salzes. Es ist ein weifser, in Wasser etwas löslicher Niederschlag; aus welcher Lösung es in kleinen Warzen oder auch in schön weifsen Krusten krystallisirt, die sich am Licht schnell färben.

$0,4003 \mathrm{~g}$ bei $90^{\circ}$ gotrocknet hinterliofsen beim Gltiben $0,1265 \mathrm{Ag}$.

$$
\begin{array}{ccc} 
& \text { Berecbnet ftir } & \text { Gefanden } \\
\mathrm{Ag}_{\mathrm{g}} & \mathrm{C}_{6} \mathrm{H}_{10} \mathrm{NCO} . \mathrm{C}_{8} \mathrm{H}_{4} \mathrm{COOAg} & \\
\hline 1,76 & \mathbf{3 1 , 6 2 .}
\end{array}
$$

Das piperilenphtalaminsaure Piperidin :

$$
\mathrm{C}_{6} \mathrm{H}_{4}<\mathrm{COOH} . \mathrm{C}_{8} \mathrm{H}_{10}
$$

schmilzt, in einem offenen Kölbchen in dem Schwefelsăurebad erhitzt, gegen 150\%. Bei höherer Temperatur zerlegt es sich theilweise in Piperidin und Piperilenphtalaminsăure. Die Elimination von Wasser und also seine Umwandlung in Piperilenaminphtalein gelingt, wenn man das Piperidinsalz im goschlossenen Rohr, oder besser bei Gegenwart eines wasserentziehenden Salzes erhitzt. Mischt man es zu dem Endo mit wasserfreiem Strontiumchlorid, so tritt die Reaction gegen 200" ein. Man zieht das Product mit Aether aus, wäscht es mit Kali, unterwirf es ron neuem der Behandlung mit Aether und erhält nach Vertreibung desselben das Piperilenaminphtaleïn mit allen seinen charakteristischen Bigenschaften. 
Dibrnmpiperilenaminphialeïn. - Zur ätherischen Lósung des Phtaleins fügt man tropfenweise eine ätherische Bromlösung. Anfänglich bildet sich ein schweres röthliches Oel, das bei langsamem weiteren Hinzufügen von Brom vollständig in glänzenden, zu Gruppen vereinigten Prismen krystallisirt. Diese krystalle wurden mehrmals mil Aether gewaschen und dann über Schwefelsäure getrocknet.

Bei Behandluug mit Kalilösung gahen sie sich als ein Bromadditionsproduct des Phtaleïns zu erkennen.

Die Brombestimmung wurde nach der von Hugo Schiff modificirten Piri a 'schen Methode und schliefslich durch Titriren nach Volhard's Wethode ausgeführt.

I. $0,1046 \mathrm{~g}$ variangton $4,35 \mathrm{cbcm}$ Normalsilberlosung $=0,0348 \mathrm{Br}$.

II. $0,1262 \mathrm{~g}$ aus Alkohol umkrystallisirte rhombische, etwas gefärbte Tafein reriangten 4,9 cbom Normalsilberlbsung $=$ $0,0392 \mathrm{Br}$.

$\overbrace{\mathrm{Br}} \frac{\text { Berechnet für }}{\mathrm{C}_{8} \mathrm{O}_{2}\left(\mathrm{~N}, \mathrm{C}_{5} \mathrm{H}_{20}\right)_{2}+2 \mathrm{Br} ; \mathrm{C}_{8} \mathrm{H}_{4} \mathrm{O}_{2}\left(\mathrm{~N}, \mathrm{C}_{4} \mathrm{H}_{10}\right)_{2}+4 \mathrm{Br}} \underset{51,61}{\overbrace{34,7}} \underset{39,3}{\text { Gefunden }}$

Wenn auch die Ergebnisse der Analysen nicht gut mit der Berechnung stimmen, so ist doch ersichtlich, dafs die dargestellte Substanz das Additionsproduct des Phtaleïns mit 2wei Atomen Brom ist und dafs es schon bei der Krystallisation aus Alkobol eine geringe Zersetzung erlitt.

Nach den publicirten Bestimmungen Rob. Schiff's vereinigt sich die jetzl als piperilenphtalaminsaures Piperidin erkannte Verbindung mit vier Atomen Brom, während das Phtaleïn, das durch Wasserentziehung daraus derivirt, nur zwei Bromatome bindet.

Conilenphtalantnsäure.

Die Einwirkung von Phtalsäureanhydrid auf Coniin ist analog derjenigen auf Piperidin.

Die beiden Substanzen vereinigen sich unter Wärmeent- 
wicklung und bein Erkalten entsteht eine gelbe harte tuoreacirende Masse, die kaurn den Geruch des Coniins hat.

Bei Anwendung gleicher Molecule Auhydrid und Coniin entstelt conilenphtalaminsaures Coniin, wàlirend ein Theil des Phtulsäureanhydrids unangegriffen bleibt. Dieses Salz bildet eine harzartige, nicht krystallinische Masse, die man noch direster erhäl, wenn man 2 Mol. Coniin in Alkohol auflöst, 1 Mol. Phtalsäureanhydrid zufügt und auf dem Wasserbad eindampn.

Lōst unan das so gewonnene Salz in wãsserigem Alkohol und neutralisist die lösung mit Salzsäure, so erhäh man durch Abkuhlung eine Menge von farb!osen Nadeln, in Warzen vereinigı, die uns denselben Lösungsnittel unkrystallisirt werden.

Diese Krystalle bestehen aus Conilenphtylaminsäure.

0,239; \& gaben $0,6135 \mathrm{CO}_{3}$ und $0,1645 \mathrm{H}_{2} \mathrm{O}$.

\begin{tabular}{|c|c|c|}
\hline & $\begin{array}{c}\text { Berechuet für } \\
\text { BOr:OC,H,CON } \text { C }_{w} \mathrm{H}_{1}\end{array}$ & Gefunden \\
\hline S & 39,82 & 69,83 \\
\hline $\mathbf{H}$ & $\pi, 63$ & 7,62 . \\
\hline
\end{tabular}

Die Conilenphtalamiusäure schmilat bei 155". Eben dargestellt, besteht sie aus farblosen, schwach glänzenden Krystallen, die sich mit der Zeit bräunen und opak werden. Beim Umkrystallisiren aus siedende!n wässerigem Alkohol setzt sie sicb theilweise in suures phtulsaures Coniin um, welches nicht trystallisirt. Sie ist wenig in Aether und Wasser löslich, Hiwas mehr in Benzin, leicht in wässerigem Ammoniak, aus dem sie nach Zusatz von Salzsäure wieder auskrystallisirt. Das Ammoniaksalz dieser Sãure giebı mit Baryumchlorid keinen Niederschlag, wohl aber nit essigsaurem Blei und mit Silbernitral solche von weifser Farbe, dic wenig oder gar nicht in Wasser löslich sind.

Conilomphcalaminsaures $\boldsymbol{K}^{\prime} u p f$ for. - Man erhüll dieses 
Salz miltelst essigsauren Kupfers als ein hellgrũnes Pulver, das in Wasser wenig löslich ist.

$0,1845 \mathrm{~g}$ bei $100^{\circ}$ getrocknet gaben beim Glthen $0,025 \mathrm{CaO}$.

$$
\begin{aligned}
& \text { Berechnet für Gefunden } \\
& \left(\mathrm{C}_{8} \mathrm{H}_{18} \mathrm{~N}, \mathrm{CO} . \mathrm{C}_{6} \mathrm{H}_{4}, \mathrm{CO}_{8}\right)_{2} \mathrm{Cu} \\
& \mathrm{CuO} \\
& 13,23 \\
& 13,55 \text {. }
\end{aligned}
$$

Conilenaminphtaloin.

Das conilenphtalaminsaure Coniin :

$$
\mathrm{C}_{6} \mathrm{H}_{4} \prec \mathrm{COOH} \cdot \mathrm{NC}_{8} \mathrm{H}_{16} . \mathrm{C}_{8} \mathrm{H}_{16}
$$

in einem geschlossenen Gefäfs bis auf $200^{\circ}$ erhitzt, verwandelt sich nicht in das entsprechende Phtalein. Erhitzt man aber bei einer höheren Temperatur, z. B. auf einem Asbestbad oder in offenem Kolben, so wird Wasser frei und zu gleicher Zeit destillirt auch etwas Coniin.

Das entstehende Product ist gefärbt. Man behandelt es zunächst mit concentrirter Kalilauge und zieht es dann mit Aether aus; den Rückstand der ätherischen/Lösung wäscht man mit Salzsäure und nimmt ihn wieder mit Aether auf.

Der ätherischen Lösung entzieht man schliefslich die Salzsäure, welche sie enthält, durch Schütteln mit eimigen Tropfen Kalilösung.

Nach Entfernung des Aethers bleibt das Conilenphtalein in der Form eines dicken, fast ungefärbten Oels zurück, das schwach nach Coniin riecht. Vollständig trocken bildet es eine feste, amorphe, wenig fluorescirende Masse.

Zur Analyse wurde das Phtalein auf $80^{\circ}$ erwärmt und in Vacuum bis zu constantem Gewicht getrocknet.

$0,304 \mathrm{~g}$ gaben $0,8357 \mathrm{CO}_{2}$ und $0,254 \mathrm{H}_{2} \mathrm{O}$.
Berochnet für
C $\mathrm{C}_{8} \mathrm{H}_{4} \mathrm{O}_{2}\left(\mathrm{~N}, \mathrm{C}_{8} \mathrm{H}_{16}\right)_{2}$
H 75,0
Gefunden
9,37 74,97 9,31 .

Das Conilenaminphtaleïn ist in den gewoobnlichen Lñsungs- 
milteln leicht löslich. Es löst sich auch leicht in einer kalten wässerigen Auftösung von Kali oder Ammoniak, aus der es beim Erwärmen ausfältt; beim Erkalten wird es fest. Concentrirte Kalilauge verändert es auch in der Wärme nicht; nur beim Schmelzen mit Kali zerfällt es in Coniin und Phtalsäure.

Ich konnte dieses Phtaleïn trotz Anwendung verschiedener Lōsungsmittel und Bedingungen nicht in krystallinischer Form erhalten.

Bromconilenaminphtaleïn. - Brom in ătherischer Lösung reagirt auf Conilenaminphtaleīn in gleicher Lōsung unter Entstehung eines rōthlich gefärbten Additionsproducts, das nicht krystallisirt und allmählich Brom verliert. Diese Verbindung entspricht wahrscheinlich derjenigen, die das Piperilenaminphtaleïn unter gleichen Bedingungen bildet.

Bisher hatte man azotirte Phtaleïne nicht erhalten, mit Ausnahme der Diphenylverbindung L ell mann's, deren Natur aber nicht erkannt worden war und einer mit dem Phtalimid isomeren Verbindung, die Kuhara beschrieb ${ }^{*}$ ), aber selbst nicht wieder darstellen konnte.

Diese azotirten Phtaleine leiten sich direct von den Salzen ab, welche die bezüglichen Phtalaminsäuren mit den entsprechenden Basen bilden. Für diese Säuren und Salze können folgende allgemeine Formeln nicht in Zweifel gazogen werden :

$$
\begin{aligned}
& \text { für die Säure } \mathrm{C}_{6} \mathrm{H}_{6}<\mathrm{COOH} \mathrm{NR}_{2}, \\
& \text { für das Salz } \mathrm{C}_{6} \mathrm{H}_{4}<\mathrm{COOH} \cdot \mathrm{NR}_{2} \\
& \mathrm{CONR}
\end{aligned}
$$

Würde die Elim ination von Wasser aus diesen Salzen

-) Bull. 89, 519 . 
auf die gewölnliche Weise erfolgen, wie dies bei den Salzen der Amine der Fall ist, so würden symmetrische Diamide der Phtalsäure entstehen von der allgemeinen Formel :

$$
\mathrm{C}_{6} \mathrm{H}_{4} \backslash \mathrm{CO} . \mathrm{NR}_{2}
$$

Phtalsäurediamide dieser Art sind bis jetzt gänzlich unbekannt, ebenso wie das normale Diamid der Phtalsäure.

Andererseits unterscheiden sich normale Diamide in ihren charakteristischen Eigenschaften merklich von den in dieser Mittheilung als azotirte Phtaleine beschriebenen Verbindungen.

Während die normalen Amide der Säuren fast alle gut krystallisiren, hoben wir unter diesen Abkömulingen Verbindungen, die durchaus nicht krystallisiren wollen.

Die Diamide werden von einer mebr oder weniger warmen Kalilauge, die nicht einmal sebr concentrirt zu sein brauchh leicht angegriffen, die azotirten Phtaleine hingegen widerstehen auch der concentrirtesten und warmen Kalilsuge; um sie zu zersetzen, mufs man sie mit dieser Buse schmelzen.

Schon diese Eigenschaften lassen erkennen, dafs man es nicht mit normalen Amider zu thun hat, und dazu kommt, dals man für eine dieser Verbindungen, für dus Diphenylaminpintalein, die Constitution von den Dichlorphtalyl ableiten kann, für das die asymmetrische Formel jetat allgemein angenommen ist.

Um die Bildung dieser Verbindungen zu erklären; muls man annohmen, dafs das Wasserstoffatom der Sâure nebat demjenigen des secuudüren Amins ein Sauerstoffatom in der Form von Wasser eliminiren, and dufs dieses Sauerstuffatom nicht vom Carboxyl der Phtalaminsăure, sondern vom Carbonyl genommen wird, das schon einen Rest des Monamins bindet. So murs nothwendigerweise der einworthige Rest des als Base functionirenden Monanins oine der zwei frei werdeuden Valenzen des Kohlenstods sättigen, whend die ondere Yalen, 
sich an den Sauerstoff bindet, welcher dann die beiden Kohlenstoffatome in derselben Weise vereinigt, wie im Phtat säureanhydrid :

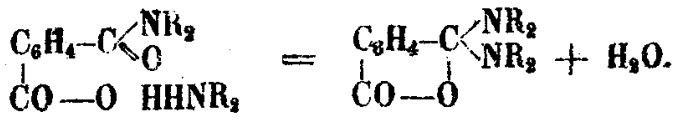

Nach diesen Betrachtungen über die Constitution dieser azotirten Phtaleine gebe ich in Folgendon eine Vebersicht derselben, zusanmen mit den Säuren, von weichen sie abgeleitet werden können :

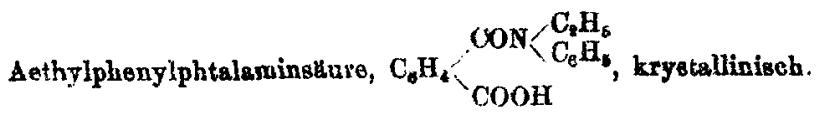

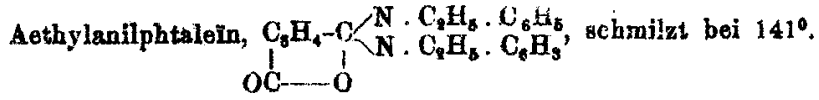

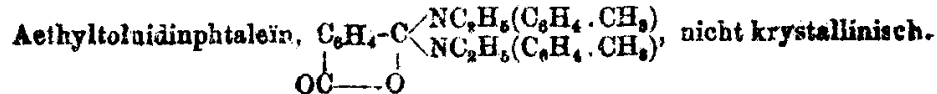

Diphenglphtalaminature, $\mathrm{C}_{6} \mathrm{H}_{4}<\mathrm{COOH}\left(\mathrm{COH}_{6} \mathrm{H}_{3}\right)_{9}$, solmilat bei $149^{\circ}$.

Diphenylaminphtalein, $\mathrm{C}_{6} \mathrm{H}_{4}-\mathrm{C}<\left(\mathrm{C}_{8} \mathrm{H}_{5}\right)_{3}$, schmilat bei $288,6^{\circ}$.

Piperilenphtalaminshurs, $\mathrm{C}_{6} \mathrm{H}_{4} \div \mathrm{COOH} \cdot \mathrm{CO} \cdot \mathrm{C}_{3} \mathrm{H}_{10}$, Atäsaig.

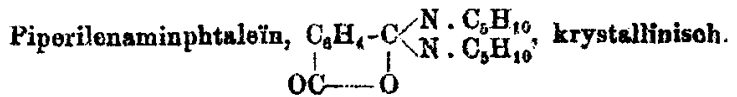

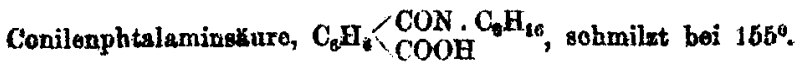

Conilonaminphtalein, $\mathrm{C}_{8} \mathrm{H}_{4}-\mathrm{C}\left\langle\mathbf{N} \cdot \mathrm{C}_{8} \mathrm{H}_{48}\right.$, nicht krystallinisch. or: -0

Orthotolylphtalimid.

Kommen wir jelzt auf die Producte der Einwirkung von Phtalsäureanhydrid auf den ersten bei 203 bis $209^{\circ}$ siedenden Theil der genischten Aethylaniline zurück. Ich exhielt neben 
206 Piutti, Einwirkung von Phtalsdureanhydrid u.s. 2.

dem Phenylphtalimid eine in weifsen Warzen krystallisirende. gegen $17^{\circ}$ schmelzende Substanz, welche in Alkohol reichlicher als das Phenylphtalimid löslich war.

Diese Substanz verhielt sich gegen Kalilauge wie ein substituirtes Phtalimid, indem sie damit eine flüssige flüchtige Base und Phtalsãure gab.

0,266 g Substanz gehben 0,7362 $\mathrm{CO}_{3}$ and $0,1138 \mathrm{H}_{3} \mathrm{O}$.

Diese Werthe stimmen mit den für die Formel des Tolylphtalimids :

$$
\mathrm{C}_{6} \mathrm{H}_{4}<\left\langle\mathrm{CO}^{\mathrm{CO}}>\mathrm{N}\left(\mathrm{C}_{6} \mathrm{H}_{4}, \mathrm{CH}_{8}\right)\right.
$$

berechneten überein.

$\begin{array}{ccc} & \text { Berechnet } & \text { Gefunden } \\ \text { C } & 75,95 & 75,77 \\ \text { H } & 4,64 & 4,76 .\end{array}$

Leh hatte schon bei der Zersetzung der Phtalamidobenzoësăure mit $p$-Toluidin das $p$-Tolylphtalimid erhalten, das in langen, gegen $201^{\circ}$ schmelzenden Nadeln krystallisirt; das jetzt analysirte Tolylphtalimid mufs also die o-Verbindung sein, da die $m$-Verbindung schon dadurch ausgeschlossen ist, dafs sich im käuflichen Anilin kein $m$-Toluidin findet.

Ich wollte nichts desto weniger $\mathrm{zu}$ besserem Beweise dieses Tolylphtalimid durch directes Ausgehen vom 0-Toluidin darstellen. Die so gewonnene Substanz zeigte sich nach allen Beziehungen als identisch mit dem o-Tolylphtalimid, das ich bei der oben besprochenen Reaction erhalten habe.

Prof. Schiff's Laboratorium in Florenz. 